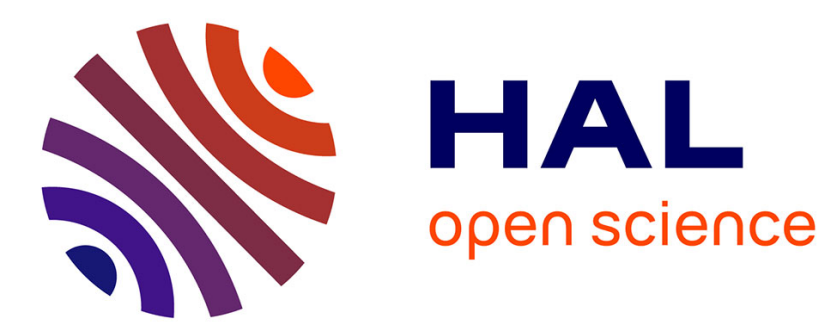

\title{
Passion de l'ignorance
}

Alain Vanier

\section{- To cite this version:}

Alain Vanier. Passion de l'ignorance. Cliniques méditerranéennes, 2004, Haïr, ignorer, 2 (70), pp.59 66. $10.3917 / \mathrm{cm} .070 .0059$. hal-01522351

\section{HAL Id: hal-01522351 \\ https://hal.science/hal-01522351}

Submitted on 6 Jun 2017

HAL is a multi-disciplinary open access archive for the deposit and dissemination of scientific research documents, whether they are published or not. The documents may come from teaching and research institutions in France or abroad, or from public or private research centers.
L'archive ouverte pluridisciplinaire HAL, est destinée au dépôt et à la diffusion de documents scientifiques de niveau recherche, publiés ou non, émanant des établissements d'enseignement et de recherche français ou étrangers, des laboratoires publics ou privés. 


\section{Alain Vanier}

\section{Passion de l'ignorance}

Il y a, semble-t-il, un lien traditionnel et évident entre l'ignorance et la passion. En faisant de l'ignorance une passion, passion de l'ignorance, Lacan ne fait-il que confirmer cette articulation ou fait-il un pas de plus si l'on veut bien entendre cette passion de l'ignorance aux deux sens du génitif ? Mais commençons par un commencement :

«Telle fut, Athéniens, l'enquête qui m'a fait tant d'ennemis, des ennemis très passionnés, très malfaisants, qui ont propagé tant de calomnies et $\mathrm{m}^{\prime}$ ont fait ce renom de savant. Car, chaque fois que je convaincs quelqu'un d'ignorance, les assistants s'imaginent que je sais tout ce qu'il ignore. En réalité, citoyens, c'est probablement le dieu qui le sait, et, par cet oracle, il a voulu déclarer que la science humaine est peu de chose, ou même qu'elle n'est rien ${ }^{1}$. »

Socrate mène une enquête parce que la Pythie de Delphes a déclaré que nul n'était plus savant que lui, ce qui l'a profondément troublé. Il s'acharne dès lors à dissoudre cette fausse réputation, entendant rencontrer tous ceux qu'il suppose être plus savants que lui. Et dans ce que Socrate dit ici à ses juges, on trouve ce lien entre la passion et l'ignorance, mais aussi la mort, car on sait que cette affaire se termine mal.

Le renom de savant et ce rapport particulier au savoir - « chaque fois que je convaincs quelqu'un d'ignorance » on s'imagine que « je sais tout ce qu'il ignore »- mais aussi, c'est " le dieu qui le sait », ce qui pourrait s'articuler avec les questions du sujet moderne : on pourrait commenter chacun des mots que Platon met dans la bouche de Socrate, pour faire jouer les

Alain Vanier, psychanalyste, ancien psychiatre des hôpitaux, professeur à l'université Paris 7 - Denis Diderot ; 47, avenue Paul Doumer, F-75116 Paris.

1. Platon, Apologie de Socrate, trad. M. Croiset, Paris, Les Belles Lettres, 1920. 
usages que Lacan fera de cette passion de l'ignorance, parce qu'il y a des usages de cette notion chez Lacan, et non pas un usage.

Il y a, certes, une grande polysémie de ce terme de passion, polysémie qui rend parfois assez difficile de savoir précisément de quoi l'on parle. Cette polysémie est - Lacan l'indique - non seulement actuelle, mais aussi historique, car nous entendons ce terme de passion après et avec toute une tradition philosophique qui a lié diversement, mais toujours, passion et ignorance. On peut se demander si la passion est un concept analytique, mais pour l'ignorance, nul doute que Lacan lui ait donné ce statut. Roland Gori souligne cela dans son très beau livre, et ce que je vais essayer de vous dire va recouper ce qu'il a pu avancer ${ }^{2}$.

Ce que nous entendons par passion est sans doute assez éloigné de ce que les Anciens ont voulu dire, d'autant que «l'ignorance consolidée », qui fut celle des grandes années de la psychiatrie a fait passer les passions au rang de catégorie nosographique. Ce fut l'œuvre de de Clérambault, et un tel passage n'est pas rien ; on pourrait, ici, en appeler à Foucault. Donc, une polysémie diachronique, historique.

« Ignorance consolidée » est un terme de Lacan. Il suggère d'ailleurs qu'il y a un type d'ignorance différent suivant les époques et les cultures. Il est donc utile de faire jouer cette sorte d'imprécision et de faire travailler les termes qui font le titre de cette communication.

Plus tard après un certain tournant historique, la passion apparaît comme fondamentalement une manifestation de l'ignorance. Ainsi, Descartes pensait que les hommes ne connaissent pas les passions et ne cherchent pas à s'en délivrer. Pour lui, les passions de l'âme sont les passions des pensées que l'âme ne dirige pas. Pour pouvoir se gouverner, il convient de les connaître et savoir que ce sont des pensées qui viennent du corps, causées par les mouvements du corps et non par une autre âme, maligne, qui nous habiterait à notre insu ${ }^{3}$. On pourrait, là aussi, détourner ces formulations à notre profit afin d'y retrouver un certain nombre de propositions de la psychanalyse, ce qui se conçoit assez bien.

Il existe une tradition occidentale qui a toujours lié la passion à la méconnaissance. La psychanalyse s'y inscrit. Le patient qui vient nous voir, sujet à ces passions, celui qui souffre, qui pâtit, souffrirait de cette méconnaissance qu'est l'inconscient. Faire savoir serait le remède, et lui pas plus que l'homme de Descartes ne cherche à s'en délivrer. Notre patient sait sans savoir ce savoir. Mais pour Freud, ce qu'il ne sait pas, c'est que c'est selon son vœu.

2. Roland Gori, Logique des passions, Paris, Denoël, 2002.

3. Voir Descartes (1649), Les passions de l'âme, Paris, Gallimard, 1953. 
L'ignorance est avant tout méconnaissance, marque du premier niveau de division, qui distingue le moi et le sujet, aussi bien que le conscient de l'inconscient. Bien que l'existence de l'inconscient freudien constitue un écart par rapport à cette tradition, le projet freudien au début, la prolonge.

Pourtant, l'inconscient est une complication qui n'est pas sans conséquences et ce sont celles-ci que je voudrais très, trop rapidement, approcher. Lacan nous a fourni, me semble-t-il, les outils pour interroger ce mouvement. Ce que je vais essayer de faire valoir part d'une remarque presque triviale.

Commençons par le commencement, le transfert. Lacan propose en 1954 le schéma d'un dièdre à six faces ${ }^{4}$.

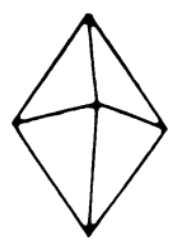

Pour ce que j'ai à vous dire, on peut ne retenir que la moitié de cette figure, un polyèdre à trois faces, c'est-à-dire une pyramide. L'une de ces faces correspond au Symbolique, l'autre à l'Imaginaire et la troisième au Réel. À la jonction de ces trois registres, se trouvent les arêtes passionnelles qui se manifestent dans le transfert. "Un tel schéma vous présentifie ceci - c'est seulement dans la dimension de l'être, et non pas dans celle du réel, que peuvent s'inscrire les trois passions fondamentales - à la jonction du symbolique et de l'imaginaire, cette cassure, si vous voulez, cette ligne d'arête qui s'appelle l'amour - à la jonction de l'imaginaire et du réel, la haine - à la jonction du réel et du symbolique, l'ignorance. » Et Lacan ajoute : "L'amour et la haine ne vont pas sans cette troisième, qu'on néglige, et qu'on ne nomme pas parmi les composantes primaires du transfert - l'ignorance en tant que passion. Le sujet qui vient en analyse se met pourtant, comme tel, dans la position de celui qui ignore. Pas d'entrée possible dans l'analyse sans cette référence - on ne le dit jamais, on n'y pense jamais, alors qu'elle est fondamentale. » Il y a lieu d'insister sur « se met », sur la façon dont le dispositif conduit le sujet à se mettre dans une certaine position pour que l'analyse s'engage. L'ignorance se place à la jonction du réel et du symbolique.

4. Jacques Lacan (1953-1954), Les écrits techniques de Freud. Le séminaire, Livre I, texte établi par J.A. Miller, Paris, Le Seuil, 1975, p. 297-298. 
À juste titre, Roland Gori a insisté sur le lien entre cette ignorance propre à l'analyse et la règle fondamentale qui fonde cette ignorance et lui donne statut par anticipation ${ }^{5}$. Pour Lacan, à cette époque, le procès de l'analyse consiste en l'édification progressive de la pyramide supérieure - d'où le polyèdre à six faces - où « l'être se réalise. » Ainsi « ces trois passions sont les voies de la réalisation de l'être. »

Lacan commente ensuite l'amour et la haine, insistant sur l'ambivalence et leur mutation respective. Pour l'ignorance, c'est un peu différent, une double valence. "Si le sujet s'engage dans la recherche de la vérité comme telle, c'est parce qu'il se situe dans la dimension de l'ignorance - peu importe qu'il le sache ou pas. C'est l'un des éléments de ce que les analystes appellent readiness to the transference, ouverture au transfert. Il y a chez le patient ouverture au transfert du seul fait qu'il se met dans la position de s'avouer dans la parole, et chercher sa vérité au bout, au bout qui est là, dans l'analyste. Chez l'analyste aussi, il convient de considérer l'ignorance. » L'analyste n'a pas, lui, à " guider le sujet sur un Wissen, un savoir, mais sur les voies d'accès à ce savoir [...] En d'autres termes, la position de l'analyste doit être celle d'une ignorantia docta, ce qui ne veut pas dire savante, mais formelle, et qui peut être, pour le sujet, formante. » Il ajoute : " La tentation est grande parce qu'elle est dans l'air du temps [et je trouve qu'elle l'est plus que jamais], de ce temps de la haine, de transformer l'ignorantia docta en ce que j'ai appelé, ce n'est pas d'hier, une ignorantia docens. Que le psychanalyste croie savoir quelque chose, en psychologie par exemple, et c'est déjà le commencement de sa perte. "

Je vous propose maintenant de faire un saut dans le temps, et de considérer ce que Lacan présente vingt ans plus tard où, là aussi, joue entre les registres un certain type $\mathrm{d}^{\prime}$ intersection 6 .

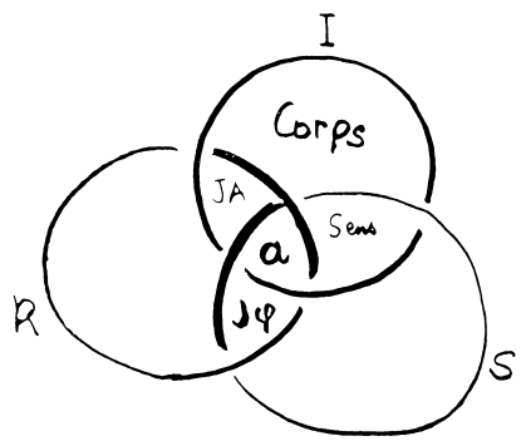

5. Voir Roland Gori, Logique des passions, op. cit.

6. Jacques Lacan (1974), « La Troisième », Lettres de l'École freudienne, 1975, n 16. 
L'inconscient de 1974 n'est plus tout à fait celui de 1954. Dans ce nœud borroméen, chaque rond représente l'une des dimensions - Imaginaire, Symbolique et Réel - à l'intersection desquels se trouvent, entre l'Imaginaire et le Symbolique, le sens, entre l'Imaginaire et le Réel, la jouissance de l'Autre, et, entre le Réel et le Symbolique, la jouissance phallique.

Le sens est à l'intersection de l'Imaginaire et du Symbolique à la jonction desquels se situe l'arête de l'amour dans le schéma de 1954. Il y a un lien, Lacan l'a indiqué, entre le sens et l'amour qui s'incarnent dans la religion, dont il n'est pas nécessaire de souligner la fortune dans notre culture. Il y a aussi une proximité, à l'intersection de l'Imaginaire et du Réel, de la jouissance de l'Autre et de la haine, cette haine que déclenche la jouissance que présentifie l'Autre pour le sujet. Enfin, à l'intersection du Réel et du Symbolique, il y a la jouissance phallique, là où se trouvait l'arête de l'ignorance, et l'ignorance, le mouvement entre l'ignorance et le savoir, est quelque chose du mode de manifestation de la jouissance phallique dans le transfert. La jouissance phallique est la marque de l'ignorance, d'un autre niveau de l'ignorance que celui que Lacan avait avancé en 1954.

L'ignorance se présente comme passion dans le transfert, et masque une ignorance d'un autre registre. Il y a autant de niveaux d'ignorance que de niveaux de la division subjective : un premier niveau, déjà évoqué, puis celui que Lacan nomme refente. Si quelqu'un s'adresse à nous, c'est qu'il fait l'expérience de l'ignorance où le laisse son symptôme, et qu'il pense, suppose que le savoir peut y remédier. Il y a une dimension historique dans le statut de l'ignorance. Ce n'est pas une idée, qui va de soi de tout temps, que le savoir peut remédier à la souffrance. Lacan peut dire : « Le symptôme est l'irruption de cette anomalie en quoi consiste la jouissance phallique, pour autant que s'y étale, s'y épanouit ce manque fondamental que je qualifie du non rapport sexuel. » La jouissance phallique, en quelque sorte, témoigne paradoxalement de cette béance fondamentale, elle témoigne de cette ignorance structurale fondamentale au cœur du savoir.

Cette ignorance située se trouve du côté de l'analyste. Est-ce qu'on peut encore la dire docte ? Ce n'est pas évident, et d'ailleurs, dans ces années-là, Lacan ne va plus s'appuyer sur cet aspect de la philosophie occidentale, quant au statut des passions et de l'ignorance. On ne peut plus la dire docte au sens de Nicolas de Cues, car, pour cela, il fallait une certaine époque. On pourrait soutenir, comme Lacan le fait à propos de l'histoire de la psychiatrie, que chaque époque organise son mode d'ignorance, tout comme chaque époque organise ses modes et ses canaux de jouissance, et son mode spécifique de malaise. Et c'est en cela que l'ignorance est liée au savoir. Il reste sans doute à écrire une histoire de l'ignorance. 
Ce n'est pas dans le champ de la philosophie occidentale, mais dans le bouddhisme, que Lacan dit avoir trouvé la passion de l'ignorance parce que le bouddhisme est le seul qui considère l'ignorance comme une passion. Ce n'est pas du tout la même chose de considérer l'ignorance comme une passion que de penser que nous ignorons nos passions parce que leurs causes nous sont dissimulées. Dans le bouddhisme, il y a deux niveaux d'ignorance - surtout dans le bouddhisme zen, celui qui intéressait Lacan. Il a un premier niveau où l'ignorance est conjuguée à la soif - envie ou désir. Et cette conjugaison de l'ignorance et de la soif ou du désir est la cause de toutes les passions, la cause du mal. Dans le bouddhisme zen, il y a une sorte de paradoxe : au-delà d'une ignorance pourrait-on dire originaire, existe aussi semble-t-il un autre niveau de l'ignorance qui fait de l'ignorance quelque chose d'absolument identique à ce que les bouddhistes zens appellent l'éveil. L'ignorance et l'éveil, ou même ignorance de l'ignorance, à ne pas entendre simplement comme négation de négation, c'est l'éveil. La psychanalyse comme pratique de réveil, avancée par Lacan dans ces années-là trouve ici un écho.

La passion de l'ignorance peut alors se comprendre autrement. " L'inconscient, ce n'est pas que l'être pense [...] l'inconscient, c'est que l'être, en parlant, jouisse, et, $[\ldots]$ ne veuille rien en savoir de plus [...] ne veuille rien en savoir du tout » parce que la sexualité est au centre de tout ce qui se passe dans l'inconscient, et qu'elle y est comme manque, comme défaut fondamental ${ }^{7}$. Nous ne voulons rien savoir de ce qui peut se savoir, précisément pour ne pas savoir ce qui ne peut pas se savoir. Nous ne voulons pas savoir là où le savoir bute; c'est même ce qui fait le succès des religions.

Ce quelque chose d'irréductible est ce qu'une certaine opération constitutive de notre ignorance produit et nous laisse ignorants de la seule vraie passion qui nous habite, qui est la passion du signifiant, qui ne se manifeste jamais plus nettement que dans la psychose. De cette passion du signifiant, dans la névrose, le phallus fait signe, mais, en même temps, voile. Le névrosé vit la passion du signifié, du phallique comme signification. Quelque chose fait joint, plus ou moins adéquat, entre le corps et le langage. C'est l'effet de l'opération constitutive du signifié phallique, qui génère la signification que nous ne cessons de produire, opération qui a des vertus modératrices. On peut ainsi concevoir un lien métonymique entre les différentes passions qui les fait s'équivaloir, voire donne une sorte de préséance à l'ignorance même, comme témoignant d'une mise en place de la structure en tant que condition du transfert.

7. Jacques Lacan (1972-1973), Encore, Le Séminaire, Livre XX, texte établi par J.-A. Miller, Paris, Le Seuil, 1975. 
Je voudrais terminer par deux extraits d'histoires cliniques, qui m'ont beaucoup appris sur un certain statut de l'ignorance au début du traitement. Elles proviennent des premiers temps de ma pratique, et montrent deux positions de l'ignorance dans cette sorte d'opération initiale dans la cure qui consiste, pourrait-on dire, à situer l'ignorance. Il s'agit d'un jeune homme qui vient depuis peu de temps. Il possède une certaine information sur la psychanalyse du fait de sa profession. Il manque une séance, et apporte un rêve à la séance suivante, qu'il interprète soigneusement après en avoir fait le récit. De lui avoir simplement fait remarquer que ce rêve venait à la place de la séance manquée a provoqué une surprise qui a marqué une bascule en modifiant le régime de sa parole et a constitué le début de sa cure. C'est ainsi, me semble-t-il, que le psychanalyste introduit, soutient, répond de l'hypothèse de l'inconscient, c'est-à-dire situe l'ignorance, ignorance de ce savoir qu'est l'inconscient, et la fait apparaître autrement. L'autre brève histoire clinique concerne une jeune femme qui consulte pour un sentiment diffus d'insatisfaction conjugale et quelques difficultés professionnelles. Après quelques entretiens, elle me fait le récit suivant. Ce matin-là, en prenant son bain, elle s'est masturbée avec le jet de la douche. Un peu plus loin dans la séance, elle me dit qu'elle a réfléchi. Elle peut payer ses séances et assurer les dépenses quotidiennes de la famille. Mais elle devra renoncer aux dépenses, quelques babioles, des vêtements, qu'elle fait quand elle a " un coup de blues ». Il me semble que là aussi, est situé quelque chose de ce qui peut se savoir dans une analyse, de ce qui se sait sans se savoir sur la jouissance. À cela, il y a une limite, et le mouvement du traitement cerne un «non-savoir qui n'est pas ignorance » ou une ignorance d'un autre niveau, corrélat du savoir produit dans la cure.

Je n'ajouterai qu'une chose pour conclure : l'ignorance a d'abord désigné, au XIVe siècle « l'état d'une personne qui n'est pas avertie des réalités de la vie, et en particulier de la vie sexuelle ${ }^{8} »$. Je n'ai rien voulu dire d'autre.

\section{Résumé}

En faisant de l'ignorance une passion, Lacan faisait plus que de reconduire le lien traditionnel qui veut que la passion soit une manifestation de l'ignorance. Avec la notion de savoir insu, la psychanalyse change le statut de l'ignorance et la situe comme passion du transfert, avec l'amour et la haine. L'ignorance témoigne alors d'un fait de structure fondamental qui la relie à la jouissance phallique. 
Mots-clés

Amour, haine, ignorance, jouissance, passion, savoir, transfert.

THE PASSION OF IGNORANCE

Summary

By making ignorance into a passion, Lacan was doing more than renewing the traditional link that tells us that passion is a manifestation of ignorance. With the notion of knowledge " unbeknown ", psychoanalysis changes the status from ignorance and posits it as passion of transference, with love and hate. Ignorance then bears witness to a basic structural fact that links it to the phallic orgasm.

Keywords

Love, hate, ignorance, orgasm, passion, knowledge, transfer. 